\title{
Erratum to: "Impact of age on survival in radioiodine refractory differentiated thyroid cancer patients"
}

In the above-named article by Saie et al. (European Journal of Endocrinology 2021184 (5) 667-676; (https://doi.org/10.1530/ EJE-20-1073)), incorrect Figure 2 was published on page 672. The correct Figure 2 is published below.
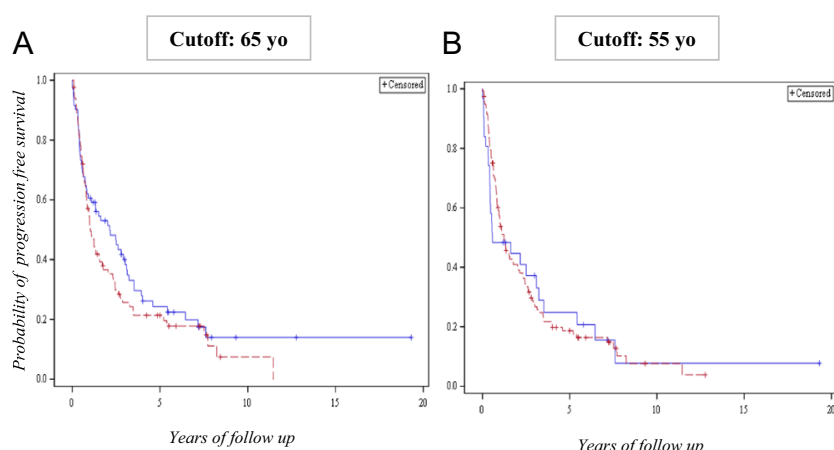

C

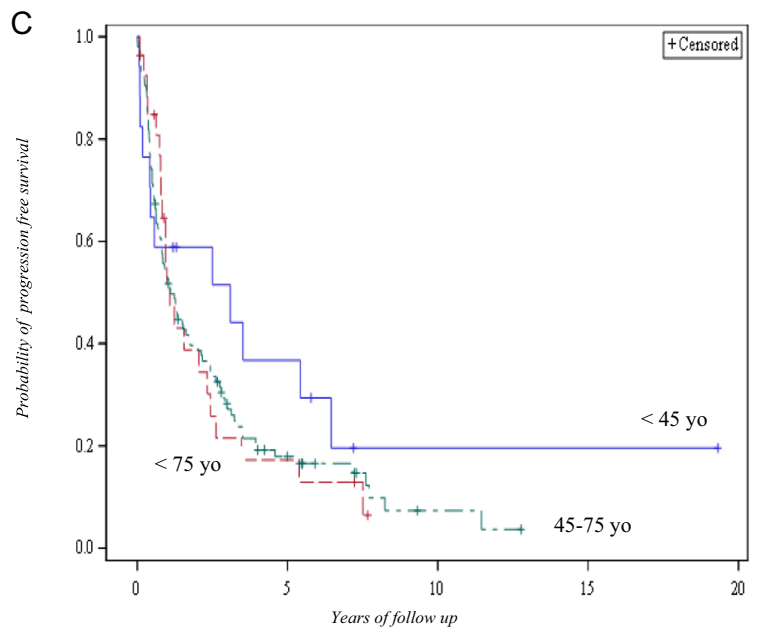

(c) 2021 European Society of Endocrinology Printed in Great Britain

\section{Figure 2}

Progression-free survival after RAIR-DTC diagnosis according to age. (A) Median progression free survival after RAIR-DTC diagnosis with a cutoff at 65 years old. Younger patients $(n=71)$ median overall survival: 2.1 years (95\% Cl: $0.8-3)$. Older patients $(n=83)$ median survival: 1.0 years $(95 \% \mathrm{Cl}$ : $0.8-1.55) . P=0.22$ between age groups. (B) Median progression free survival after RAIR-DTC diagnosis with a cutoff at 55 years old. Younger patients $(n=31)$ median overall survival: (95\% Cl: 0.2-6.5). Older patients $(n=117)$ median survival: 1.1 years $(95 \% \mathrm{Cl}$ : 0.8-1.6). $P=0.25$ between age groups. (C) Median progression-free survival after RAIR-DTC diagnosis with a cutoff at 45 and 75 years old. Younger patients ( $n=18$ ) median overall survival: 3.1 (95\% Cl: 0.2-6.5). Older patients $(n=131)$ median survival: 1.1 years $(95 \% \mathrm{Cl}$ : $0.78-2.33$ ). $P=0.25$ between age group, $P=0.77$. Blue line: $<45$ years old patients; red line: over 75 -year-old patients; green line: $45-75$ years old patients. A full color version of this figure is available at https://doi.org/10.1530/EJE-20-1073.

Published by Bioscientifica Ltd 